\title{
Embryology of some congenital cardiac anomalies
}

\author{
E. W. T. MOR R IS
}

From St. Thomas's Hospital Medical School, London

The purpose of this paper is to propose explanations for the nature of some commonly occurring congenital cardiac anomalies in terms of human embryology. The basic difficulty in any such attempt is that, in the course of the development of the human heart, there are two stages. During the first, the embryological process results in the formation of a four-chambered heart separated by an interatrial and interventricular septum into a right and left part; during the second, the organ so formed grows in size, and changes occur in the form and proportions of its component parts to achieve those of the definitive heart. As Grant (1962) points out, little is known about the second stage. In congenital heart disease, since the heart has to function continuously so long as it is alive, errors of form occurring in the first period are likely to influence changes occurring in the second. These latter may be such as to render difficult the elucidation of those errors of formation in the first stage which is the object of this paper.

The views put forward are based on the dissection of 25 infant hearts which showed such abnormalities (see Table). This study was undertaken because views still current on the aetiology of some forms of congenital heart disease are thought to be erroneous. The earlier ones (Keith, 1902, 1905) were propounded, in the light of theories then current, that ontogeny repeated phylogeny, and the abnormalities were consequently seen as arrests of development at a stage when the evolving heart resembled some phylogenetic precursor of man, such as a reptile. These views have been elaborated (Spitzer, 1923) and are included in recent clinical books (Brown, 1950 ; Taussig, 1960) and seem widely held despite recent papers (Kramer, 1942 ; Foxon, 1955) which cast doubts on them.

There is a central difficulty inherent in any attempt to explain abnormalities on the assumption that they represent a deviation to the form of a phylogenetic ancestor: no one knows, or is ever likely to know, what the hearts of the amphibian and reptilian ancestors of man were like. The assumption that they would have resembled the hearts of modern amphibians or reptiles seems open to doubt since the living orders of these two classes are, for the most part, highly specialized and therefore less likely to resemble their early common ancestor in the structure and form of their organs.

The steps in ontogeny only repeat phylogeny in a very general way. For example, in its early stages the mammalian heart resembles the fish heart in that each chamber is undivided, and the blood is impelled by it into a single ventral aorta and thence into paired aortic arch arteries. At a later stage, the common chambers become divided into a right and a left, a step that occurs in phylogeny when pulmonary respiration is established.

At the most these stages may resemble early steps in the embryology of the presumed ancestor and not the definitive heart of such a precursor. There seems little justification now for describing congenital abnormalities of the heart in terms of an atavism to the form of heart found in a modern reptile. Moreover, these explanations were mainly given at a time when the account of the development of the mammalian heart showed many gaps. Now that these have been largely filled in to give an almost complete picture it is worth while to try to explain examples of some congenital abnormalities in terms of failure either of initiation or completion of some step or steps known to occur in the normal heart.

When this has been done some abnormalities will remain which are inexplicable in terms of simple arrest: in these, some stage has not proceeded normally. In attempting to unravel what has happened, it is legitimate to try to confine the explanation to a presumed diversion of a step known to occur normally. Equally, in accordance with Ocean's dictum 'essentia non sunt multiplicanda praeter necessitatem', it is undesirable to introduce into the explanation a stage not known to occur in normal human embryology. An assumption of this sort is made by some writers (Lev and Saphir, 1945) when they postulate that, in the development of an abnormal heart, the bulbar septum is derived from ridges which normally occur in the developing heart of 


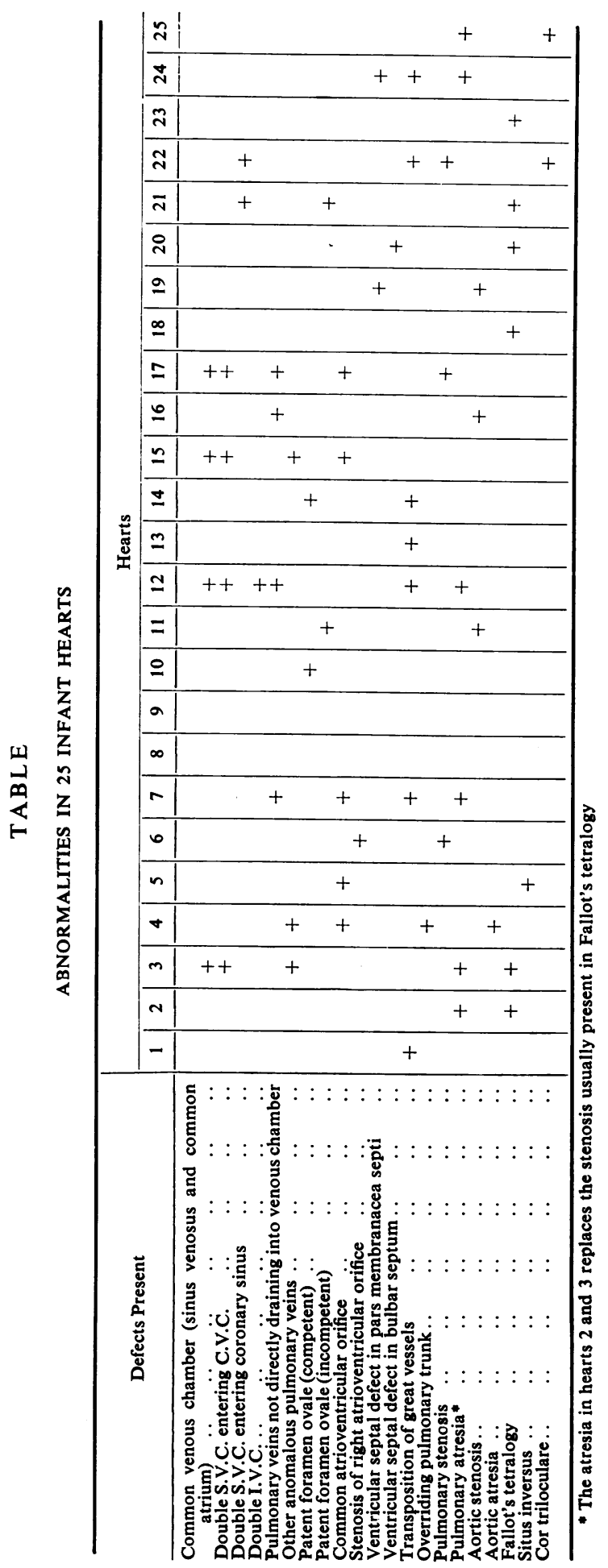


modern reptiles in order to explain overriding of the aorta and transposition of the great arteries. At no stage in the development of the human heart are there two ventral aortae arising independently from the ventricles as there are in typical modern reptiles. This is, of course, not to be confused with the fact that double aortic arches may occur as the result of the persistence of both right and left fourth arch arteries, for, when these are found, they are connected to the left ventricle by a single ascending aorta.

After some study of and thought about the specimens, various features and patterns of features displayed by them began to emerge. Even in the most complicated abnormalities it was found possible to suggest embryological explanations without invoking any steps now known not to occur in normal development. These observations and the embryological explanation for them are given below. The terms 'proximal' and 'distal' relate to the direction of blood flow through the heart.

\section{ABNORMALITIES OF THE VENTRICLE AND BULBUS CORDIS}

Reference to embryological texts (Hamilton, Boyd, and Mossman, 1962 ; Kramer, 1942 ; Odgers, 1938) shows that defects in the septation of this part of the heart can result in varying degrees of failure either of development or of union of the component parts of the septum. There are five structures or groups of structures involved in the formation of this septum; from proximal to distal, (a) the muscular interventricular septum, (b) the atrioventricular cushions forming both (i) the atrioventricular, and (ii) the interventricular parts of the pars membranacea septi, $(c)$ the proximal, and $(d)$ the distal bulbar swellings, and $(c)$ the aorticopulmonary septum.

Of these, the last to develop is the interventricular part of the membranous septum in (Odgers, 1938). If this fails to develop then the defect is found under the superior part of the septal cusp of the tricuspid valve and below the crista supraventricularis (Fig. 1) and represents a persistence of the interventricular foramen which normally exists at this stage of development. Such a defect is bounded ventrally and inferiorly by the upper border of the muscular septum, above by the bulbar septum-that is the complete septum formed from elements $(c),(d)$, 은

Abbreviations used in plates: $A W$, wall of aorta; $A S V(R)$, right septal (coronary) cusp of aortic semilunar valve; $\stackrel{\Im}{\supset}$ $A S V(L)$, left septal (coronary) cusp of aortic semilunar valve; $A S V(N)$, nonseptal (non-coronary) cusp of aortic semilunar valve; $A Z V$, azygos vein; BS, bulbar septum; $C A V(S)$, superior cusp of common atrioventricular valve; $C A V(I)$, inferior cusp of common atrioventricular valve; $C S$, crista supraventricularis; CVC, common venous chamber; IVC $(L)$, left inferior vena cava; IAS, interatrial septum; MS, muscular part of interventricular septum; $P A(L)$, left pulmonary artery; $P A(R)$, right pulmorary artery; $P M S(A)$, atrioventricular part of pars membranacea septi; PMS (I), interventricular part of pars membranacea septi; PMS(IR), rudimentary interventricular part of pars membranacea septi; $P$ TA, atretic pulmonary trunk; PTS, stenotic pulmonary trunk; $P S V(L)$, left septal cusp of pulmonary semilunar valve; $P S V(R)$, right septal cusp of pulmonary semilunar valve; $P S V(N)$, non-septal cusp of pulmonary semilunar valve; $P V$, pulmonary veins; $S V C(L)$, left superior vena cava; TVS, septal cusp of right atrioventricular valve.

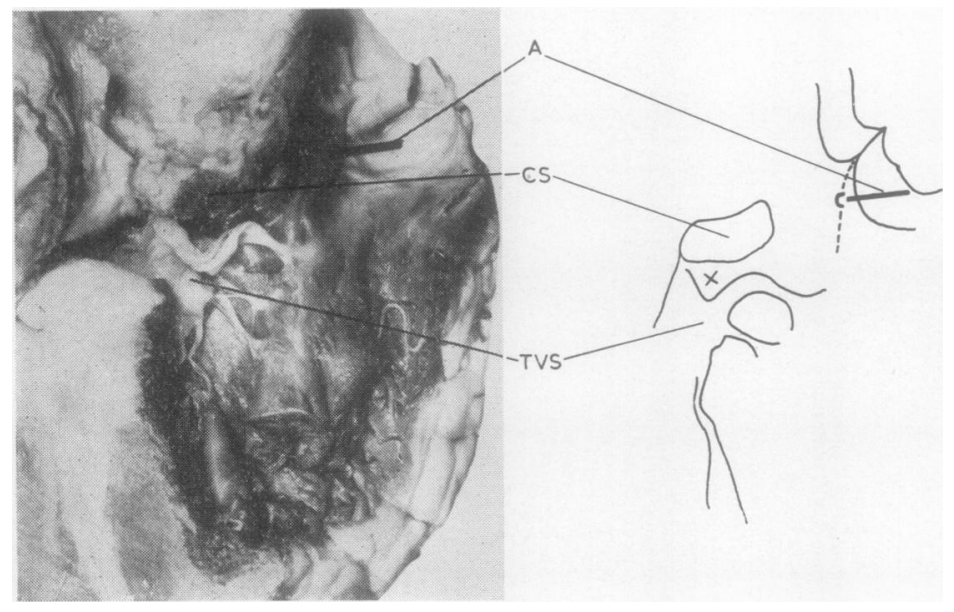

FIG. 1. Right side of interventricular septum showing position of interventricular membranous part $(x)$. Dotted line marks site of fused bulbar ridges and rod $A$ marks site of septal defect. 
and (e) already described; it is limited dorsally by the atrioventricular part of the membranous septum. This is continuous with the right wall of the aorta in the region below and between the right coronary and non-coronary cusps of the aortic valve, and has the superior part of the septal cusp of the tricuspid valve continuous with it ventrally (Fig. 2).

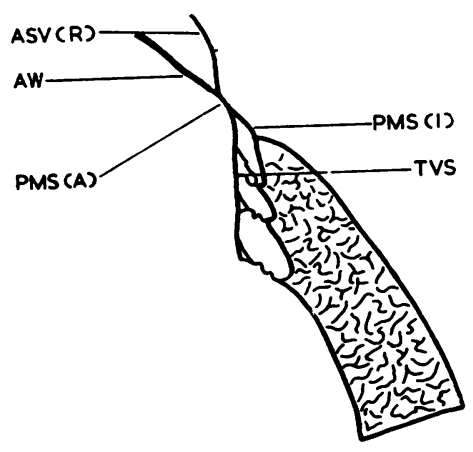

FIG. 2. Coronal section through right wall of aorta, membranous septum, septal cusp of tricuspid valve, and muscular septum.

There is in some cases (hearts $2,3,18,21$, and 23) an attempt at the formation of the interventricular part of the membranous septum which is represented by a fibrous flange in the posteroinferior part of the defect attached to the contiguous parts of the atrioventricular membranous septum and the muscular septum. It has a crescentic free border and lies to the left of the septal cusp of the tricuspid valve. It is seen in five of the hearts and is illustrated in Figures 3 and 9 .

This type of interventricular defect is usually associated with some overriding of the aorta on the muscular septum. This is hardly surprising since the normal aorta overrides the muscular part of the septum. This is due to the fact that the thin membranous septum is attached to and flush with the right surface of the thicker muscular septum, and so the upper border of the muscular septum bulges into the outflow channel of the left ventricle. Moreover, the axis of the ascending aorta is inclined to the right in relation to the plane of the muscular septum (Figs. 2 and 4). This overriding of the aorta may be accentuated even with an intact septum; this is illustrated in Fig. 5, which shows a heart (not in the series) from a dissecting-room body with a small right ventricle and pulmonary trunk due to tricuspid

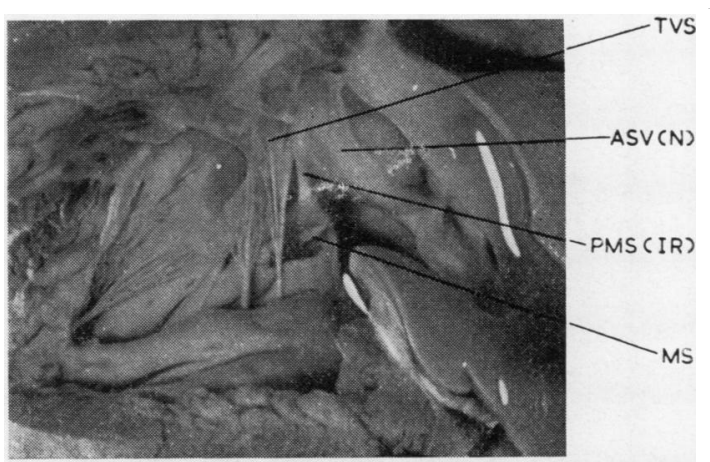

FIG. 3. Heart 2 showing aorta, septal cusp of tricuspid valve, rudiment of interventricular membranous septum, and muscular septum; a small piece has been excised from the latter.

stenosis. The aorta at the level of the semilunar valve is almost completely to the right of the plane of the muscular septum. Heart 6 in the series also shows aortic overriding with an intact septum.

When the ventricular septal defect is associated with pulmonary stenosis, as in Fallot's tetralogy, then the extent of the overriding is usually proportional to the degree of stenosis; the more extensive overriding is usually associated with a larger septal defect.

Except for mixing of blood from the two cavae in the right atrium, as soon as the atrial and

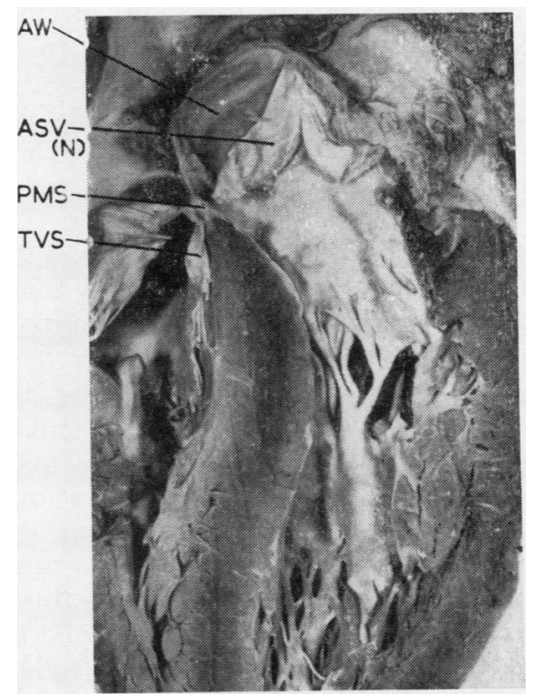

FIG. 4. Coronal section through aortic wall and interventricular septum. Compare with Fig. 2. 


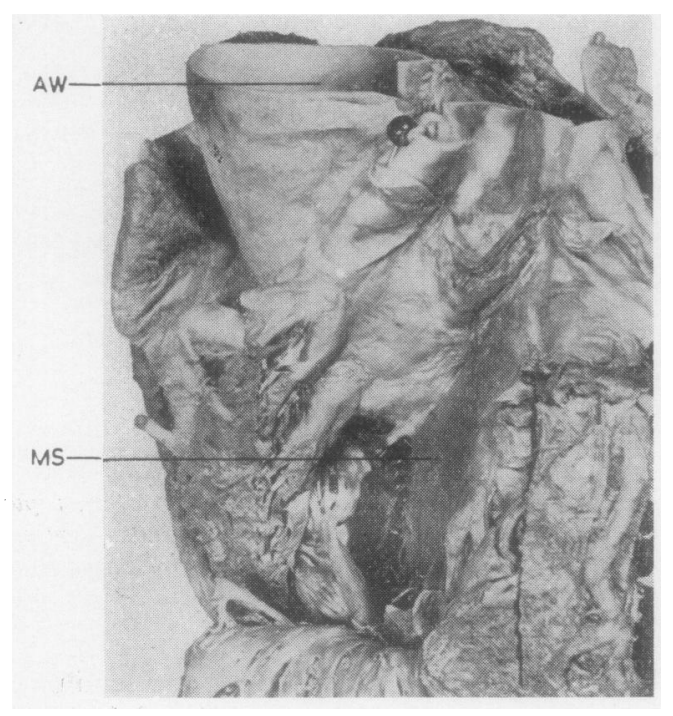

FIG. 5. View into open right ventricle and pulmonary trunk to show aorta overriding the muscular septum.

ventricular septa are complete the circulation in the embryo is divided into two separate circuits, one to the fore and the other to the hind end of the embryo, and it is the placental oxygenated blood that goes to the former. On returning from the fore end, this blood then nourishes the hind end on its way back to the placenta. This preferential treatment of the head and forelimb region is of course reflected in the relatively greater rate of growth of this part compared with the hind end during foetal life. If there is a ventricular septal defect with aortic overriding, then the blood from the right ventricle can now reach the end of the aortic arch in two ways: either via the pulmonary trunk and ductus arteriosus or via the overriding aorta. Thoma's (1893) first law suggests that there is a haemodynamic principle at work in biology according to which the calibre of a vessel is controlled by the volume of blood passing through it. Moreover, as Hughes (1943) points out, 'the dynamic relationship between vessels and circulation characteristic of the adult circulatory system is early acquired in the embryo'; once initiated, there is, in Fallot's tetralogy and allied abnormalities, a factor that reduces the flow through the pulmonary trunk, and, once initiated, this seems to be progressive and results in an enlargement of the aorta at the expense of the pulmonary channel.

In hearts (Nos. 6, 17, and 22) showing pulmonary stenosis, the pulmonary trunk, how- ever small, has always been in the same position, relative to the cusps of the aortic valve, as in the normal heart, that is adjacent to the commissure between the two coronary cusps (Fig. 6b). This suggests that in those cases the bulbus cordis has been normally divided by the distal bulbar swellings, which have also formed the semilunar valves, but that in the subsequent growth of the foetal heart the infundibulum and pulmonary trunk have failed to grow normally.

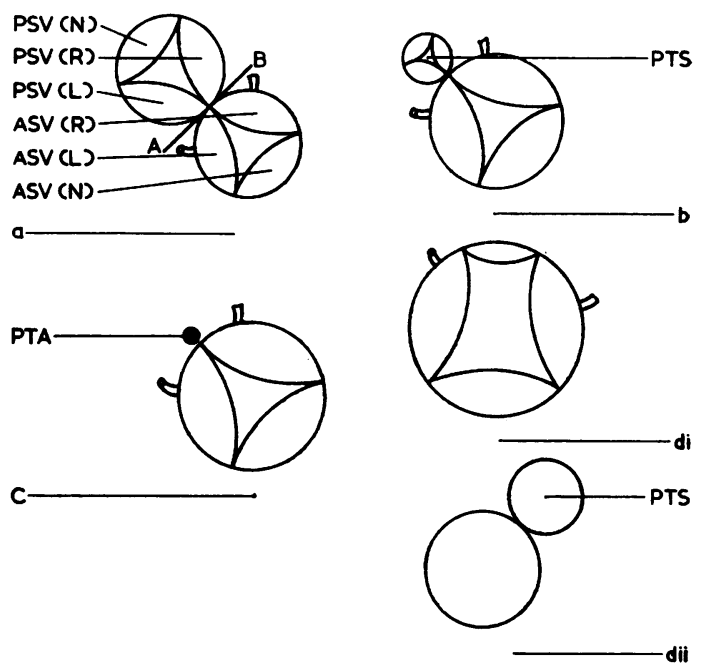

FIG. 6. Sections through the aorta and pulmonary trunk at the level of semilunar valves (except $\mathrm{d}$ ii): (a) normal, $A-B$ represents plane of bulbar septum; (b) stenosis of pulmonary trunk; (c) atresia; (d i) undivided bulbus cordis; and (d ii) just above the valve showing pulmonary stenosis.

An even more advanced stage of this process was found in several of the hearts (Nos. 2, 3, 7, 12,24 , and 25); these appear at first sight to be examples of undivided bulbus, with a single large vessel lying astride the septal defect. Further investigation, however, suggests that these are extreme cases of pulmonary stenosis with complete occlusion of the pulmonary trunk, because each one shows a small blind recess in the superior part of the right ventricle anterior to the plane of the crista supraventricularis, that is in the position of the normal infundibulum (Fig. 7). Attached to the external wall of this recess by a fibrous cord (Fig. 8) is a patent channel lying on the surface of the aortic wall and, at the level of the aortic valve (which possesses three normal cusps), it is in the position indicated in Figure 6c.

Moreover, in three of the hearts (Nos. 7, 12, and 24), which show an aorta arising mainly from 


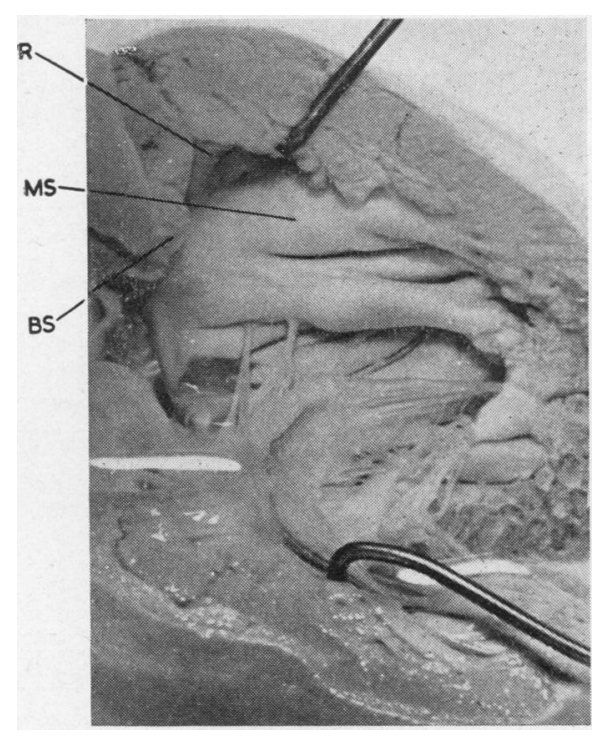

FIG. 7. Heart 2. View of the right ventricle showing infundibular recess $(R)$.

the right ventricle but with some overlap of the muscular septum, inspection of the left ventricle shows a small diverticulum between the septal. cusp of the mitral valve and the flange representing the interventricular part of the membranous septum, that is in the position of the outflow channel of the left ventricle. Arising from the external surface of the heart, corresponding to the diverticulum and dorsal to the aorta, is a fibrous cord which is continuous with a patent vessel giving rise to the right and left pulmonary arteries. These appear to be cases of transposition of the aorta and pulmonary trunk with obliteration of the proximal part of the latter. If this interpretation is correct it is interesting to note that, even when the vessels are transposed, it is the now dorsally situated pulmonary trunk that has become occluded, suggesting that some factor is operating to produce pulmonary stenosis or occlusion whatever the position of the vessel.

The hearts in this series, whatever the orientation of the great arteries relative to the sagittal plane of the body may be, all show the same orientation of the cusps of their semilunar valves relative to the other vessel, that is the arrangement of the cusps of either valve in relation to the cusps of the valve in the other channel is the same as in the normal heart. Even when the pulmonary trunk is represented by a fibrous cord, it lies adjacent to the commissure between the two coronary cusps of the aortic valve (Fig. 6c). This suggests that the division of the bulbus cordis and the formation of the semilunar valves have been normally initiated by the four distal bulbar swellings whatever has been the subsequent course of development. It seems unnecessary to postulate the occurrence in the development of these hearts of a step which is a return to the form of a phylogenetic reptilian precursor with two aortae arising from the heart. In the hearts that show transposition of the aorta and pulmonary trunk, the end product could result from an alteration in the position of the proximal and distal bulbar swellings so as to cause the pulmonary trunk to arise from the left ventricle and the aorta from the right.

It is of interest that several writers (Bremer, 1928 ; de Vries and Saunders, 1962 ; Grant, 1962) have suggested that the position of the various ridges forming the bulbar septum may be determined by a pre-existing flow of blood through the bulbus. They postulate two streams, one from the right and the other from the left part of the ventricle, flowing simultaneously through the bulbus cordis in such a way that, between the two streams, there are regions of reduced flow into which the ridges tend to grow. In the normally developing heart the position of the streams entering the bulbus is such as to cause them to spiral around each other in their course through the channel and so result in the formation of a spiral septum which rotates through approximately 180 degrees in a righthanded spiral from proximal to distal. Differences

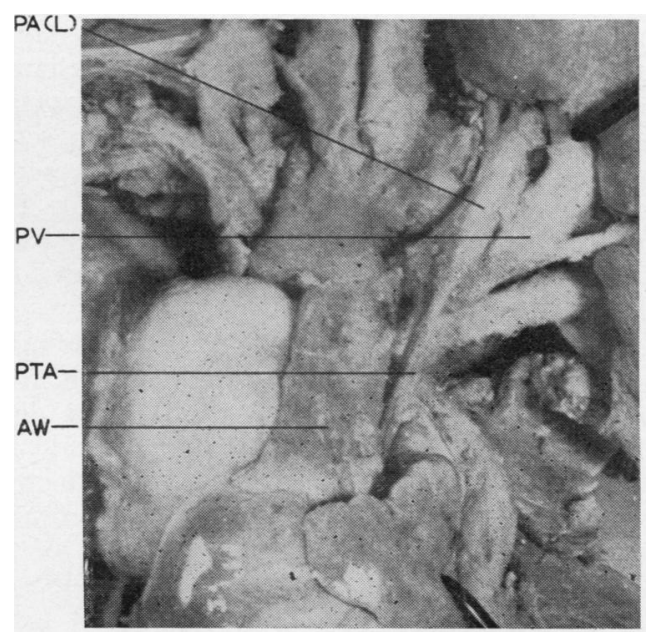

FIG. 8. Heart 7 showing proximal part of pulmonary trunk. 


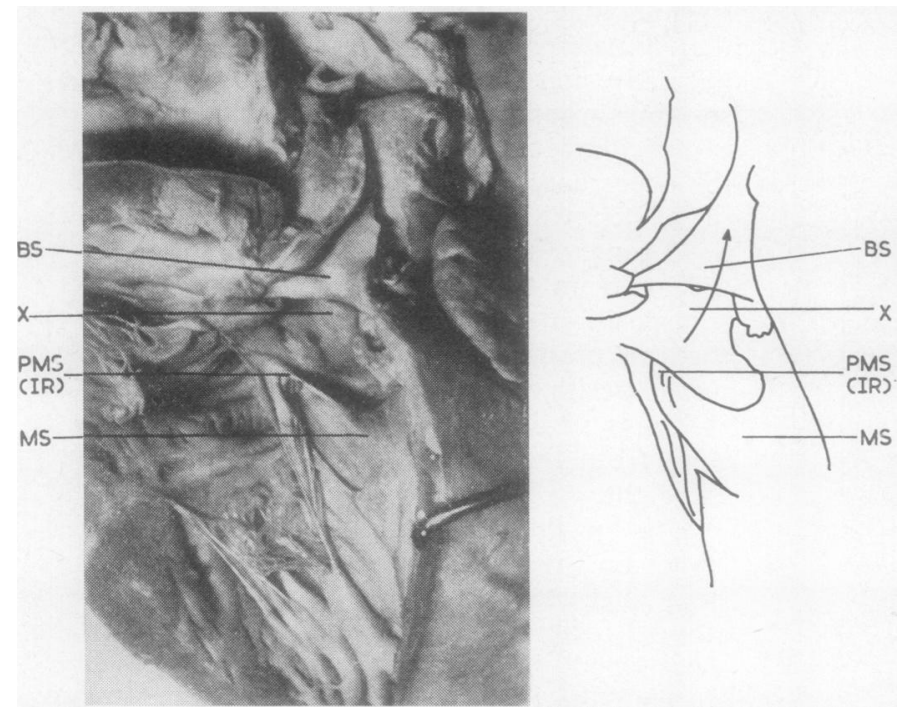

FIG. 9. Heart 20 showing compound defect in the bulbar and membranous septa throwing both outflow channels and the roots of the great vessels into communication. The arrow leads into the aorta; $(x)$ marks the posterior cusp of the common semilunar valves. (See Fig. 6, d i.)

in the relative positions of the bulbus cordis and anlagen of the right and left ventricles to each other could result in the two streams taking different courses in the bulbus and so causing the ridges and the septum derived from them to form at different sites in the bulbus cordis. This, in turn, could account for the varying degrees of transposition of the great arteries.

Although the type of ventricular septal defect that results from failure of the interventricular part of the membranous septum to develop is the commonest, others occur. One is situated just proximal to the semilunar valves in that part of the septum, formed from the bulbar ridges, which separates the distal part of the outflow channel of each ventricle (Morris, 1957). It occurs subjacent to the commissure between the two septal cusps of both aortic and pulmonary valves and is explicable as a failure of the fusion that normally occurs between the contiguous borders of the septa formed from the bulbar ridges: this defect was described by Keith (1909).

A rarer defect in the same relative position, but distal to the semilunar valves, may result from a failure of the contiguous borders of the distal bulbar and aorticopulmonary septum to unite.

One heart (No. 20) (Fig. 9) shows a more complicated defect which appzars to have resulted from a complete failure of development of the proximal bulbar ridges together with the absence of the septum formed by the distal bulbar ridges 1 and 3, although parts of the four distal bulbar swellings have formed a semilunar valve guarding the undivided proximal part of the bulbus cordis
(Fig. 6d i). Just above this valve the bulbus is divided by a septum presumably derived from the aorticopulmonary septum which has a crescentic lower free border. The pulmonary trunk so formed is much reduced in calibre (Fig. 6d ii).

Rarely, defects occur in the muscular part of the interventricular septum (Fig. 10). Such breaches cannot be explained as an arrest or retardation of any normally occurring stage in development. But, at an early stage, endothelial diverticula grow into the myoepicardial tissue constituting the ventricular wall, thus forming the trabeculae of the definitive ventricle. If the myoepicardial tissue separating two coincident

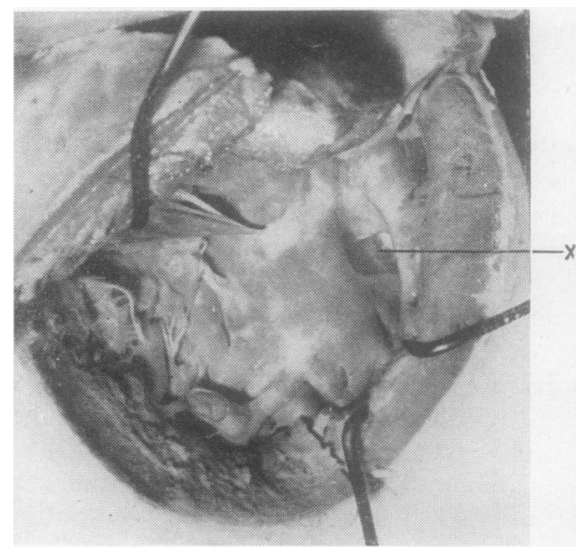

FIG. 10. Heart 8 showing defect $(x)$ in muscular part of the septum. 
diverticula growing from opposite sides of the muscular septum were to break down, such a defect would result. Keith (1909) supports such a possibility when, describing this defect, he says apparently interstices in the original muscular spongework of the septum which have remained unobliterated'. A breakdown of tissue is not unknown in the course of development; the fenestration of the dorsal mesocardium to form the definitive transverse sinus of the pericardium is an example.

ABNORMALITIES OF THE ATRIOVENTRICULAR CANAL

Another type of defect occurring in this series is a failure of fusion of the two atrioventricular cushions, thus leaving a common atrioventricular orifice (hearts $4,5,7,15$, and 17). This common orifice is transversely situated and guarded by two long cusps, attached to the superior and inferior borders of the aperture, and which therefore lie across the free border of the muscular interventricular septum (Fig. 11). The free borders of these cusps are usually attached to the muscular septum where they cross it, and at their lateral ends there may be two small cusps. These are presumably formed from the two lateral cushions of the atrioventricular canal, as the long cusps are formed from the major cushions.

The fusion of the cushions is a crucial step in the process of heart development both in position and in time. These cushions are centrally situated and involved in the formation of both the interatrial and interventricular septa, and the completion of both depends on their fusion, which is therefore anterior in time to the completion of the septa.

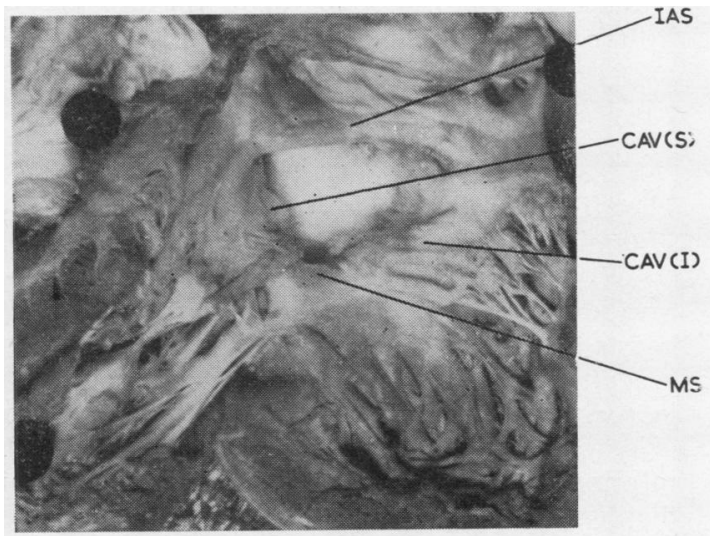

So far as the interatrial septum is concerned, if the cushions fail to fuse, the normal obliteration of the foramen primum is prevented, since this depends on fusion between the free border of the septum primum and the previously joined cushions. Moreover, since the ventral horn of the septum secundum normally grows down the fused cushions from cranial to caudal (Odgers, 1935), their failure to unite is likely to interfere with this.

In considering the effect of failure of union of the cushion on the interventricular septum, it should be remembered that, in the formation of this septum, both the bulbar and the muscular part grow towards the atrioventricular canal and the fused cushions, leaving a natural interventricular foramen which is finally closed by a growth of the inferior cushion. The cushions are therefore a focal point in the formation of the septum, and if they fail to fuse then the interventricular foramen will merge at this region with the persistent interatrial foramen primum to form a common atrioventricular defect, the boundaries of which will be, on its atrial aspect, the free border of the incomplete interatrial septum, and in the ventricular part, superiorly the free edge of the bulbar septum and inferiorly the free border of the muscular septum. In two hearts (Nos. 15 and 25) the bulbar septum as it descends the bulbus cordis has spiralled in the correct clockwise manner as seen from above so that the aorta is connected with the left ventricle and the pulmonary trunk with the right ventricle. But in two others (Nos. 7 and 12) the bulbar septum has rotated in an anticlockwise direction, resulting in transposition of the arteries with the aorta arising from the right ventricle, or right side of the

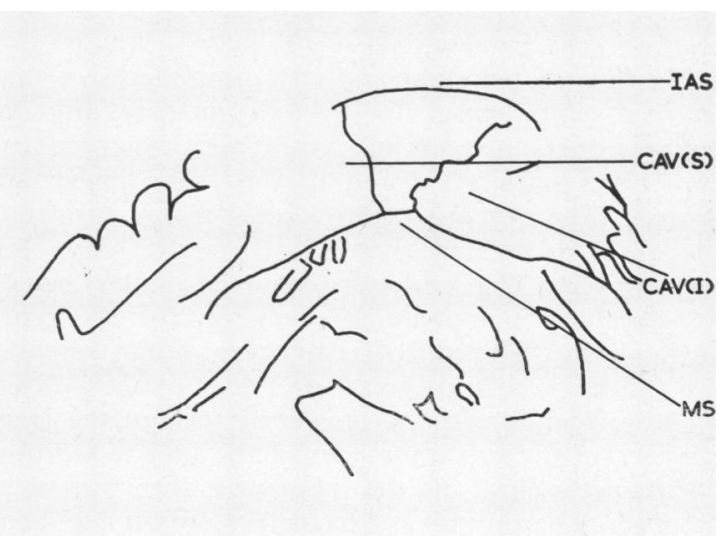

FIG. 11. Heart 15 from the left side to show the common atrioventricular defect and valve. 

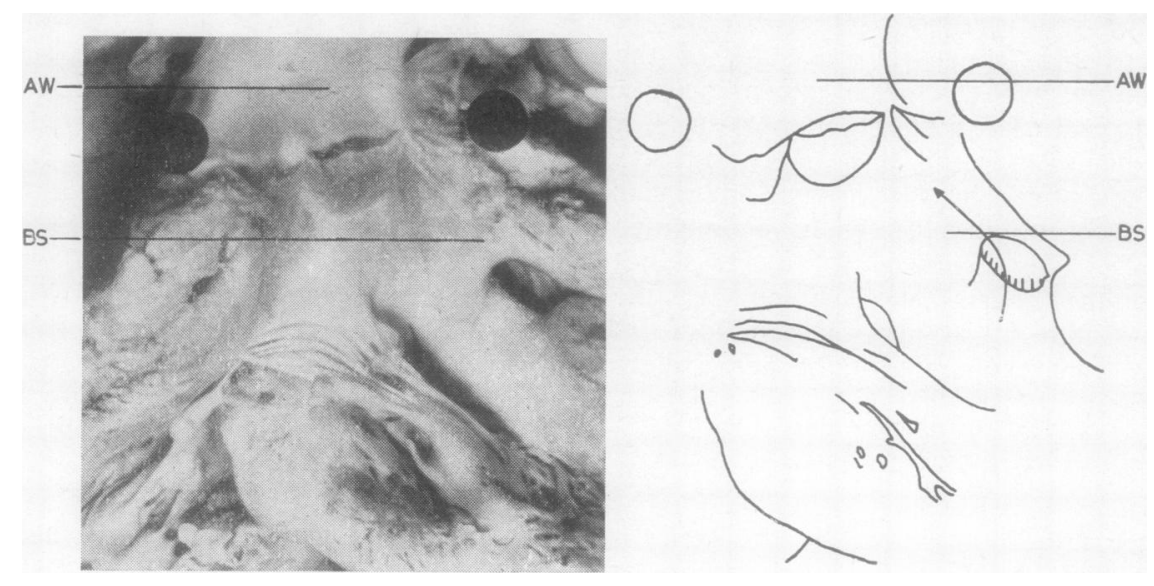

FIG. 12. Heart 17 from the right, showing common ventricle with bulbar septum fused with its wall. An arrow passes through the defect in the septum leading to a small cavity from which the pulmonary trunk arises.

common ventricle, and the pulmonary trunk, although now obliterated and represented at its beginning by a fibrous cord, arising from the left side of the ventricle.

In heart 17 the muscular interventricular septum is rudimentary, leaving the inferior border of the bulbar septum free. It is as if the bulbar ridges had grown down the adjacent wall of the common ventricle and fused together, all except for a small defect, and thus formed a small recess off the common ventricle to the left and anteriorly. One wall of this is the bulbar septum and the other is the part of the ventricular wall limited by the attachments to it of the bulbar septum. From this recess the stenosed pulmonary trunk arises superiorly. Blood could therefore pass from the common ventricle into the pulmonary trunk via the defect in the lower part of the bulbar septum (Fig. 12).

In heart 5 the right and left components of the almost completely divided atrium are mirror images of the normal in that the right side receives the pulmonary veins and the left side the venae cavae. There is an almost complete interatrial septum in which no fossa ovalis can be recognized. The common atrioventricular canal shows the arrangement of cusps usual to this type of anomaly. The muscular interventricular septum is well formed, and the anterior part of the bulbar septum blends with its superior part, but the free border of the posterior part of the bulbar septum lies to the left of the plane of the muscular septum. The pulmonary trunk lies anterior and to the left of the bulbar septum, and its origin is confined to the left ventricle. The aorta overrides the free border of the muscular septum, and its origin is between the bulbar septum and the superior cusp of the common atrioventricular valve. The arrangement of the great arteries is almost an exact mirror image of the normal. The pulmonary trunk arises in front of and to the left of the aorta and passes upwards and to the right; it continues as a patent ductus arteriosus which joins a right-sided aortic arch. The latter is joined to the heart by an ascending aorta which passes upwards and to the left behind the pulmonary trunk. This heart appears to be a case of situs inversus so far as the venous chambers, the bulbus cordis, and the aortic arches are concerned. The failure of fusion of the atrioventricular cushions has produced the same type of disturbance in the interatrial and interventricular septa as is found in the other hearts with a common atrioventricular orifice.

ANOMALIES IN THE DRAINAGE OF SYSTEMIC VEINS INTO THE HEART

The following types of abnormal drainage of systemic veins are to be found in these hearts: (1) A vein, presumed to be a left superior vena cava draining into $(a)$ the coronary sinus in two hearts (Nos. 21 and 22) and (b) opening into the left atrium or the left side of a common atrium in four hearts (Nos. 3, 12, 15, and 17) ; (2) two veins opening into the right and left sides of the inferior aspect of the atrium and draining the subdiaphragmatic part of the body (No. 12); these are presumed to be right and left inferior venae cavae. 
(1a) This condition is consistent with a persistence of the left common and anterior cardinal veins as a continuous channel. The normal fate of these veins, first described by Marshall (1850), is as follows: When the cross connexion between the two anterior cardinal veins, which forms the left brachiocephalic vein, is developed, the whole of the blood from the left forelimb and the left side of the head and neck drains via this into the right common cardinal vein and so into the right atrium. The remainder of the left anterior cardinal vein below the new channel forms the terminal part of the definitive left superior intercostal vein. The distal part of the left common cardinal vein is obliterated and forms a fibrous cord. This is connected above to the left superior intercostal vein and is continuous below with the patent proximal part of the common cardinal vein, the definitive oblique vein of Marshall, which drains into the left extremity of the coronary sinus, which is formed from the left horn of the sinus venosus.

(1b) These hearts show a vein, believed to be the left common cardinal vein, opening posteriorly into the superior aspect of the left side of the atrium (Fig. 13). This suggests that the left horn of the sinus venosus has been absorbed into the posterior wall of the left side of the atrium, leaving only the right horn to be incorporated in the right side. Such a state of affairs could occur if the constriction separating the common

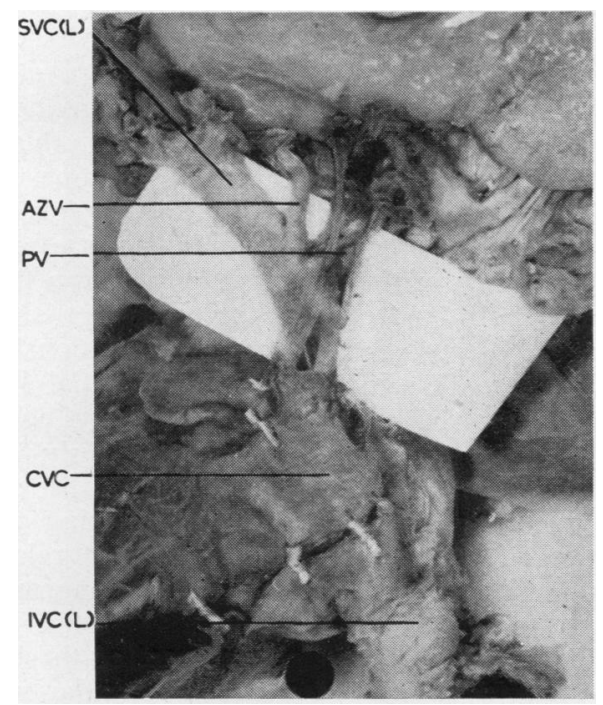

FIG. 13. Heart 12 viewed from the left, showing the left superior and inferior venae cavae. atrium from the sinus venosus, usually described as occurring at an early stage, during Streeter's Horizon $\mathrm{x}$, had been shallow and symmetrical, for it is the asymmetry of this inflexion that leads to both a reduction in the size of the sinu-atrial opening and its movement to the right side of the dorsal wall of the atrium. The venous part of the heart would then consist of an anterior common chamber, the atrium, separated from a posterior common chamber, the sinus venosus, by a shallow uniform inflexion of the wall. Moreover it is the deep indentation of the left wall that forms both the dorsal wall of the atrium to the left of the sinu-atrial opening and the anterior wall of the left horn of the sinus venosus. If this fails, the sinus venosus will be a symmetrical chamber with no horns, and the veins will open into its lateral ends. If this were the case some remnant of a left venous valve would be found in the left side of the chamber in the form of a left crista terminalis running anterior to the entrance of the left vena cava and continuing down the lateral wall of the venous chamber on the left. The chamber should be smooth-walled posterior to the cristae and with musculae pectinati arising from it anteriorly. In three of the hearts these features are present, and in one, although the venous chamber is smooth-walled posteriorly and trabeculated anteriorly and in the auricles, no crista is visible. In addition, if this theory is tenable there cannot be a normal coronary sinus, and examination shows that in none of the four hearts is it found.

(2) In this heart there are two veins opening inferiorly into the right and left sides of the common venous chamber, which are taken to be inferior venae cavae (Fig. 13). If this is correct and they have been normally derived from a right and left hepato-cardiac channel, then these should also open into the posterior part of the venous chamber, and the right one should be guarded by a normal valve continuous with the lower end of the right crista terminalis and the left one should be bordered anteriorly by a similar valve, a mirror image of that on the right. In this heart there is such a structure. In the remaining three hearts there is a single inferior vena cava.

In these four hearts it is suggested that the venous chamber is, as in the normal heart, a compound one, but, instead of the whole of the sinus venosus-both right and left horns-being absorbed into the right atrium, the symmetrical sinus venosus is incorporated into the posterior wall. The left common cardinal vein will therefore open into the left side of this common chamber and the right one into the other side. 
At the time of the initiation of the septum primum the dorsal wall of the venous chamber is therefore formed by the dorsal wall of the sinus venosus, and the dorsal mesocardium will be attached to this wall, and it is on the internal wall at this point that the septum primum normally forms. Although there is an attempt at interatrial septum formation in each of the four hearts, it is far from complete, even in two of the hearts in which the atrioventricular canal is divided.

ANOMALIES IN THE DRAINAGE OF PULMONARY VEINS INTO THE HEART

In hearts $3,4,7,12,15,16$, and 17 , the pulmonary veins do not drain normally, and in four of these (Nos. 7, 12, 16, and 17) the pulmonary veins have no direct connexion with the heart (Fig. 14). The arrangement is similar in each of the hearts in the larger group; there is a transverse channel lying behind the venous heart chamber, and into the lateral ends of this the right and left pulmonary veins drain, but this transverse channel has no connexion with the heart. The pulmonary veins are derived from the pulmonary plexus, and this is then tapped by an outgrowth from the dorsal wall of the left atrium (Auer, 1948 ; Neill, 1956). If this outgrowth failed, the pulmonary veins would be derived solely from the pulmonary plexus and would have no opening into the heart. In all the hearts with this anomaly other defects of the venous end of the heart are

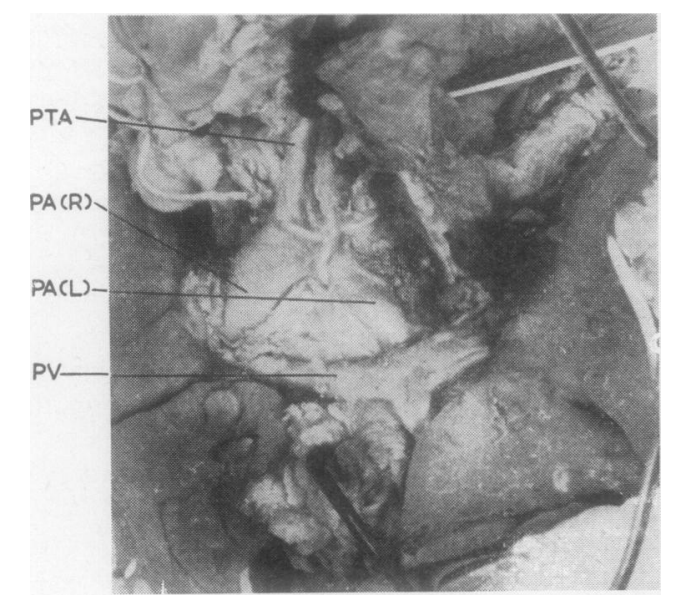

FIG. 14. Heart 12 turned upward to show the ventral surface of the lung roots. The pulmonary veins are connected by a common channel. The pulmonary trunk shows atresia proximally. present. The pulmonary plexus has numerous connexions with both the cardinal and the vitelloumbilical system of veins and, in the absence of the normal drainage via the common pulmonary vein, any of these connexions may enlarge. Numerous papers have been written on the variety of forms of this anomalous pulmonary drainage. Considering the small volume of blood that passes through the foetal lung, the size of these connexions is not important until birth and post-natal survival increase the pulmonary flow, when they must be adequate to carry this. In the three newborn hearts, the connexions are small, whereas in two others, which were from infants who survived birth by 30 hours and 12 weeks respectively, the communications between the pulmonary and other veins were well developed.

In two hearts the pulmonary veins drain into the venous chamber and presumably therefore some form of common pulmonary vein must have developed, but in each case the abnormality of double superior venae cavae is present, and so this must have grown out from the posterior wall of the sinus venosus.

\section{SUMMARY}

It has been pointed out that certain structures occurring in the normal heart can be recognized in most of the specimens. For example, all the hearts show a muscular structure, taken to be the lower border of the bulbar septum, which at one stage in development forms part of the boundary of the interventricular foramen normally existing at that stage and which then separates the outflow channels of the pulmonary trunk and aorta. Whatever the orientation of these channels relative to each other, this free border can be recognized, even when one of the orifices has become obliterated, and is represented by a recess in the ventricle. In these cases the proximal part of the vessel is replaced by a fibrous cord. Furthermore, if semilunar valves are present, the cusps bear a constant relationship to the septum. This indicates that the initial formation of the bulbar septum is a very constant feature and that, while there are obviously factors which influence the orientation and the degree and direction of twist of the developing bulbar septum, its actual formation is relatively of uninfluenced by the occurrence of abnormalities in that region of the heart.

The junction of the anterior part of the bulbar septum with the muscular interventricular septum rarely fails to take place when the latter is present. In those hearts, as in Fallot's tetralogy, in which 
the last stage of the interventricular septum-the interventricular membranous septum-has been arrested, an attempt at its formation can be observed. This takes the form of a fibrous ridge in the posterior border of the defect and bears the same relationship to the right aortic wall, the atrioventricular part of the membranous septum, and the septal cusp of the tricuspid valve as in the normal heart. Moreover this relationship persists if, owing to malrotation of the bulbar septum, it is the right wall of the pulmonary trunk to which the atrioventricular membranous septum is attached.

An explanation is offered for the occurrence of defects in the muscular interventricular septum.

When fusion of the atrioventricular cushions fails to take place, other defects also occur and these are consequent upon the earlier failure of cushion fusion.

An explanation is offered for the occurrence of a superior and inferior vena cava on the left side of the venous chamber, and it is suggested that the failure of the pulmonary veins to drain directly into the venous end of the heart has a simple embryological explanation.

The majority of cardiac anomalies seem to be compatible with an embryological explanation, and any resemblance to the hearts seen in other vertebrate classes is coincidental.

I wish to express my gratitude to Professors D. V. Davies and C. H. Barnett for help and advice and to Mr. A. Pache for the photographs.

\section{REFERENCES}

Auer, J. (1948). The development of the pulmonary veins in man. Anat. Rec., 101, 581.

Bremer, J. L. (1928). Part 1. An interpretation of the development of the heart. Part 2. The left aorta of reptiles. Amer. J. Anat., 42, 307.

Brown, J. W. (1950). Congenital Heart Disease, 2nd ed. Staples, London.

de Vries, P. A., and Saunders, J. B. de C. M. (1962). Development of the ventricles and spiral outflow tract in the human heart. Contr. Embryol. Carneg. Instn, 37, 87 [No. 256].

Foxon, G. E. H. (1955). Problems of the double circulation in vertebrates. Biol. Rev., 30, 196.

Grant, R. P. (1962). The embryology of ventricular flow pathways in man. Circulation, 25, 756.

Hamilton, W. J., Boyd, J. D., and Mossman, H. W. (1962). Human Embryology, 3rd ed. Heffer, Cambridge.

Hughes, A. F. W. (1943). The histogenesis of the arteries of the chick embryo. J. Anat. (Lond.), 77, 266.

Keith, A. (1902). The anatomy of the valvular mechanism round the venous orifices of the right and left auricles, with some observations on the morphology of the heart. Ibid., 37, proc. ii.

- (1905). Exhibition of thirty malformed human hearts. Ibid., 39, proc. xiv.

(1909). Hunterian lectures on malformations of the heart. Lancet, ii, 359, 433, 519.

Kramer, T. C. (1942). The partitioning of the truncus and conus and the formation of the membranous portion of the interventricular septum in the human heart. Amer. J. Anat., 71, 343.

Lev, M., and Saphir, O. (1945). A theory of transposition of the arterial trunks based on the phylogenetic and ontogenetic development of the heart. Arch. Path., 39, 172 .

Marshall, J. (1850). On the development of the great anterior veins in man and mammalia. Phil. Trans., 140, 133.

Morris, E. W. T. (1957). The interventricular septum. Thorax, 12, 304.

Neill, C. A. (1956). The development of the pulmonary veins with reference to the embryology of anomalous pulmonary venous return. Pediatrics, 18,880 .

Odgers, P. N. B. (1935). The formation of the venous valves, the foramen secundum and the septum secundum in the human heart. J. Anat. (Lond.), 69, 412.

- (1938). The development of the pars membranacea septi in the human heart. Ibid., 72, 247.

Spitzer, A. (1923). Uber den Bauplan des normalen und missbildeten Herzens. Virchows Arch. path. Anat., 243, 81.

Taussig, H. B. (1960). Congenital Malformations of the Heart, 2nd ed. Harvard University Press, Cambridge, Mass.

Thoma, R. (1893). Untersuchungen über die Histogenese und Histomechanik des Gefässsystems. Enke, Stuttgart. 